\title{
Sequence Impedance Modeling of Grid-Forming Inverters
}

\author{
Weihang Yan, Shahil Shah, Vahan Gevorgian, David Gao \\ National Renewable Energy Laboratory (NREL)
}

Weihang.Yan@nrel.gov

NREL/PR-5D00-80425 
店 嘈

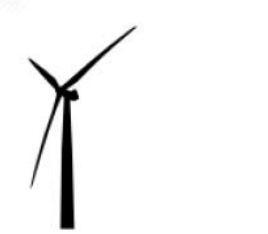

Inverter-based resources
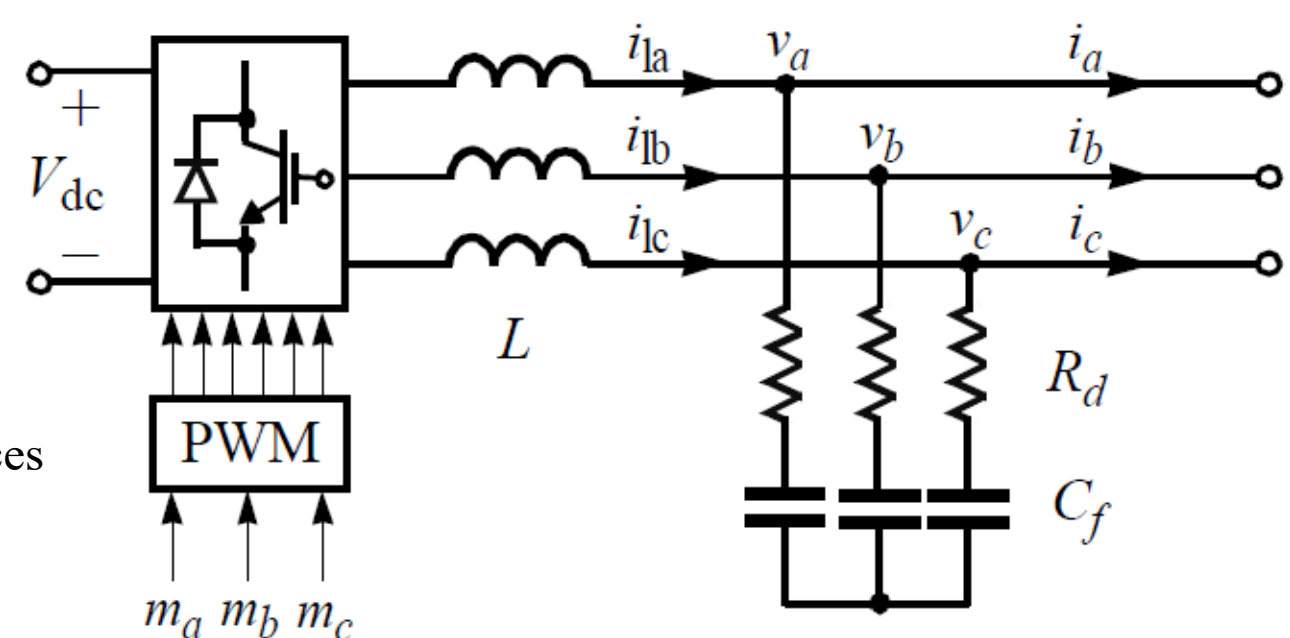

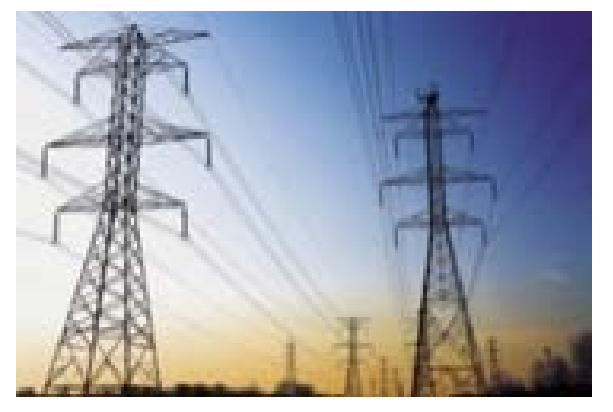

Power network
$Z_{p}(s)$
$Z_{n}(s)$

$Z_{p}(s)$
$Z_{n}(s)$

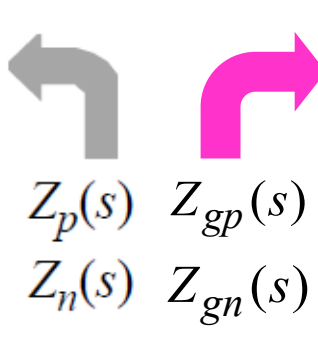

$\square$

$$
\begin{aligned}
& \stackrel{P_{\text {ref }}}{\longrightarrow} \longrightarrow D_{P} \longrightarrow D_{\omega_{1}} \rightarrow \longrightarrow \\
& \stackrel{Q_{\text {ref }}}{\longrightarrow}-D_{Q} \\
& \stackrel{v_{q, \text { ref }}=0}{\longrightarrow}
\end{aligned}
$$

- Control diagram of droop-controlled GFM inverter

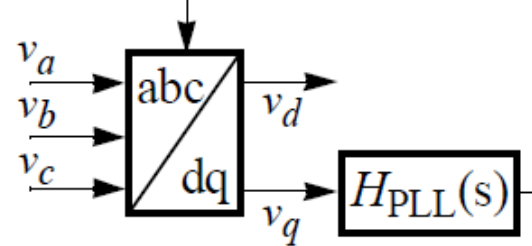

$$
\begin{aligned}
& \stackrel{\text { ref }}{\longrightarrow}
\end{aligned}
$$


Parameters of inverter controllers for GFL and GFM

\section{Control \& System Parameter}

Current inner loop crossover frequency

\section{Value}

$1200 \mathrm{rad} / \mathrm{s}$

Current inner loop phase margin/damping

$45 \mathrm{deg} / \approx 45 \%$

Outer loop crossover frequency

$120 \mathrm{rad} / \mathrm{s}$

\begin{tabular}{|c|c|}
\hline $\begin{array}{c}\text { Outer loop crossover phase } \\
\text { margin/damping }\end{array}$ & $90 \mathrm{deg} />100 \%$ \\
\hline PLL crossover frequency & $2 * \mathrm{pi}^{*} 30 \mathrm{rad} / \mathrm{s}$ \\
\hline $\begin{array}{c}\text { PL crossover phase } \\
\text { margin/damping }\end{array}$ & $45 \mathrm{deg} / \approx 45 \%$ \\
\hline Droop coefficients & $0.05 \mathrm{pu} P-\omega$ \\
\hline System operation condition & $0.05 \mathrm{pu} Q-\mathrm{V}$ \\
\hline & $P=0.85 \mathrm{MW} ;$ \\
\hline
\end{tabular}

Assumptions:

1. Frequency couplings are ignored;

2. Voltage of DC link is well controlled and considered as constant.
Time-domain simulation comparison

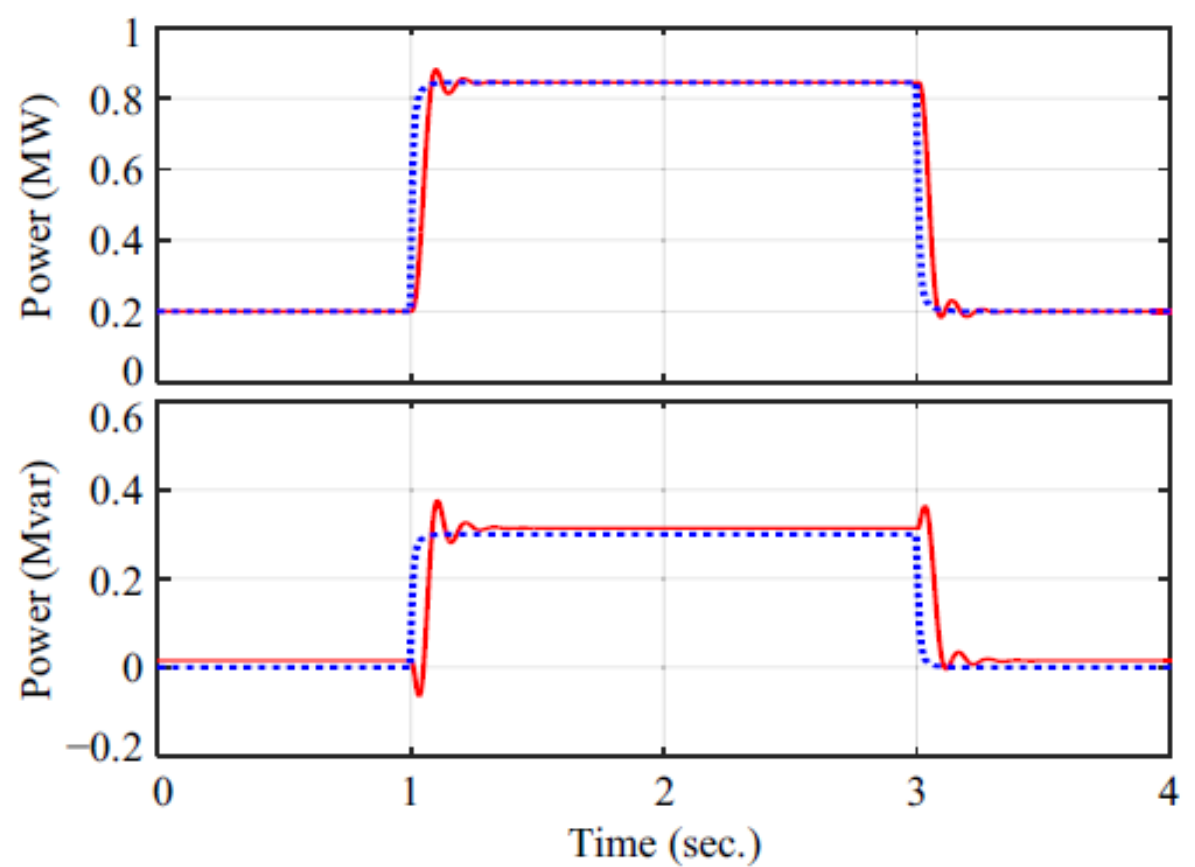

Power step change responses of GFL and GFM inverters. Blue dashed lines: GFL inverter, red solid lines: GFM inverter.

Circuit parameters for simulations

\begin{tabular}{lc}
\hline \hline Parameter & Value \\
\hline Inverter rated power & $1 \mathrm{MVA}$ \\
PCC voltage (peak), $V_{1}$ & $0.563 \mathrm{kV}$ \\
PCC current (peak), $I_{o 1}$ & $1.082 \mathrm{kA}$ \\
Inductor current (peak), $I_{l 1}$ & $1.084 \mathrm{kA}$ \\
Filter, $L, C_{f}, R_{d}$ & $3 \mathrm{mH}, 22 \mathrm{uF}, 1.87 \Omega$ \\
DC bus voltage, $V_{\mathrm{dc}}$ & $2 \mathrm{kV}$ \\
\hline \hline
\end{tabular}



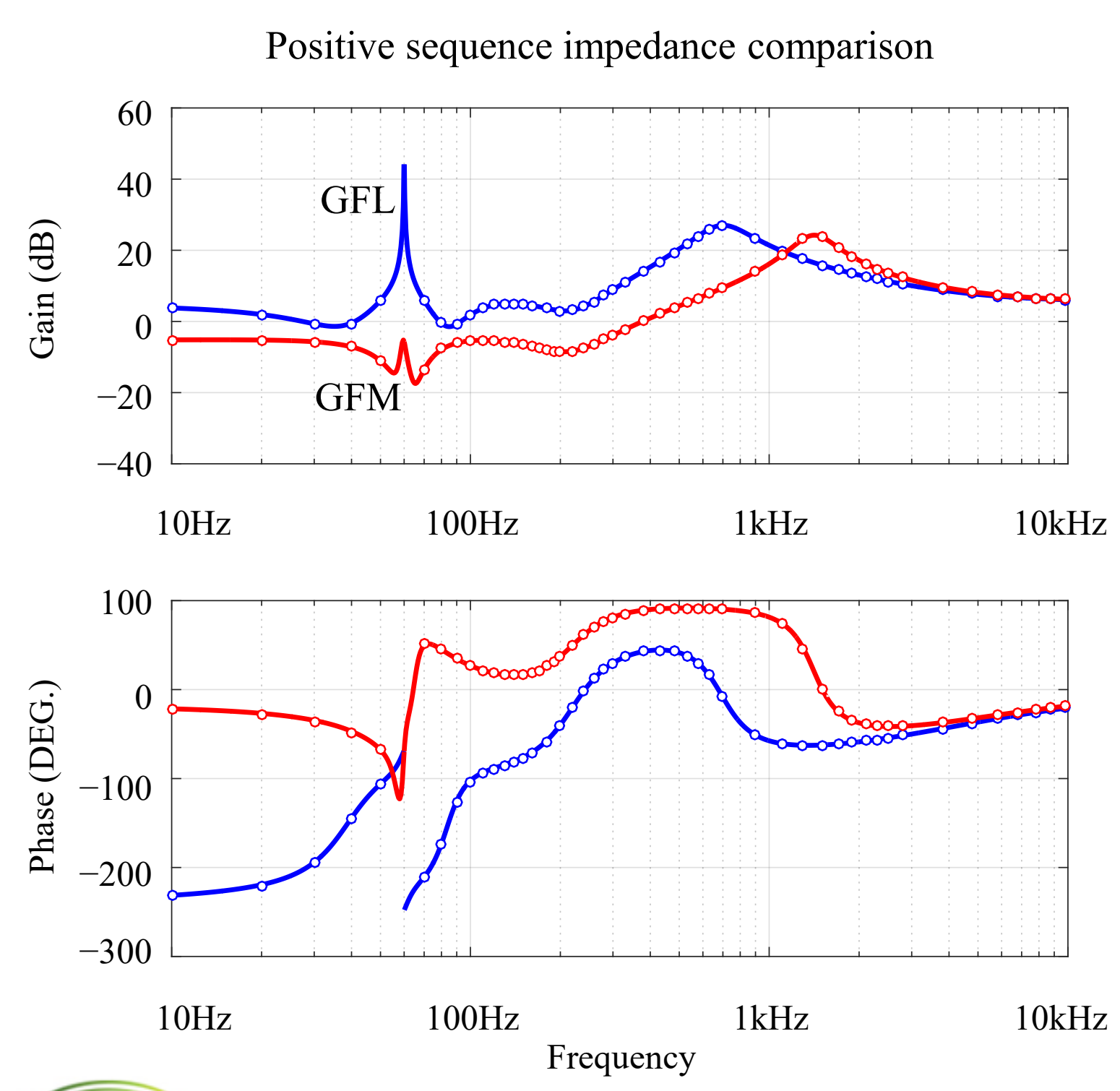

Theoretical Model $\quad$ T Simulation measurement
- GFM inverter

$$
\begin{gathered}
s L+k_{m} V_{\mathrm{dc}}\left[H_{i}\left(s-j \omega_{1}\right)-j K_{\mathrm{di}}\right] \\
+\frac{1}{2} k_{m} V_{\mathrm{dc}} V_{1}\left[K_{p}\left(s-j \omega_{1}\right)-K_{q}\left(s-j \omega_{1}\right)\right]
\end{gathered}
$$

- GFL inverter

$$
\begin{aligned}
& s L+k_{m} V_{\mathrm{dc}}\left[H_{i}\left(s-j \omega_{1}\right)-j K_{\mathrm{di}}\right] \\
& Z_{p}(s)=\frac{+\frac{3}{2} k_{m} V_{\mathrm{dc}} V_{1} G_{p}\left(s-j \omega_{1}\right) H_{p}\left(s-j \omega_{1}\right) H_{i}\left(s-j \omega_{1}\right)}{1-\frac{1}{2} k_{m} V_{\mathrm{dc}}\left\{\mathbf{I}_{l 1}\left[H_{i}\left(s-j \omega_{1}\right)-j K_{\mathrm{di}}\right]+\mathbf{D}_{1}\right\} \frac{T_{\mathrm{PLL}}\left(s-j \omega_{1}\right)}{V_{1}}} \\
& K_{p}(s)=j \frac{3}{2}\left\{V_{1}\left[H_{v}(s)-j K_{\mathrm{dv}}\right] H_{i}(s)+\mathbf{I}_{l 1}\left[H_{i}(s)-j K_{\mathrm{di}}\right]+\mathbf{M}_{1}\right\} G_{p}(s) \frac{1}{S} D_{p} \omega_{1} \\
& K_{q}(s)=j \frac{3}{2} n V_{1} H_{v} G_{p}\left(s_{1}\right)(s) H_{i}(s)
\end{aligned}
$$

- PLL is replaced with droop related terms. GFM avoids introducing negative resistance, and less likely to experience harmonic instability.

- Negative sequence impedance can be derived: $Z_{n}(s)=Z_{p}(-s)^{*}$ 


\section{Conclusion}

Impedance model of GFM inverter
- This paper presents the sequence impedance modeling of a grid-forming inverter to evaluate its small-signal stability properties.

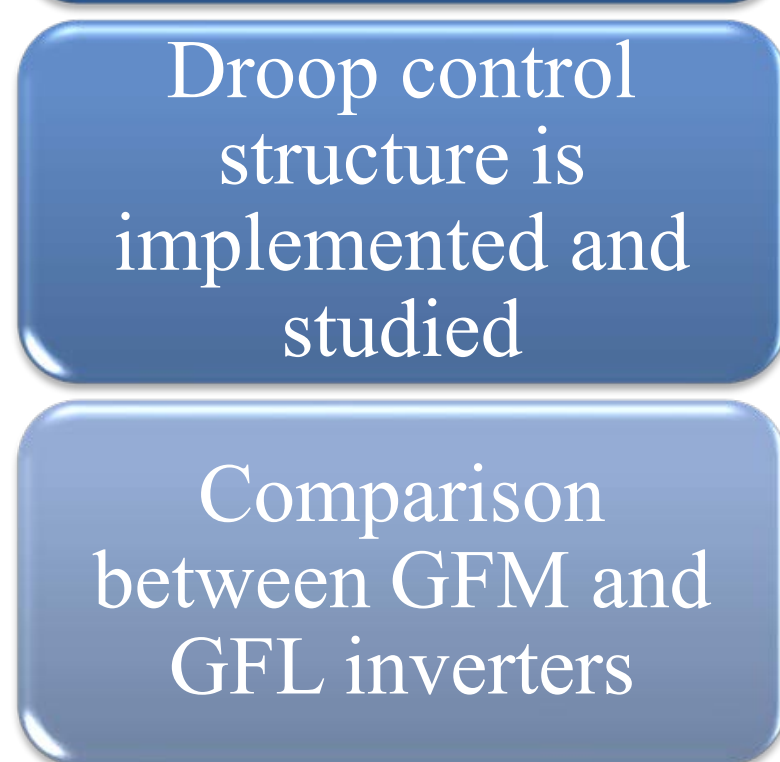

- Droop control structure is implemented to control the inverter in grid-forming mode, and the impact of individual controller on the inverter impedance characteristics is discussed.

- GFM and GFL have different grid-synchronization mechanisms, which lead to the differences of impedance models. GFM inverters is less likely to experience harmonic stability problems.

This work was authored by Alliance for Sustainable Energy, LLC, the manager and operator of the National Renewable Energy Laboratory for the U.S. Department of Energy (DOE) under Contract No. DE-AC36-08GO28308. Funding provided by U.S. Department of Energy Office of Energy Efficiency and Renewable Energy Wind Energy Technologies Office and Solar Energy Technologies Office. The views expressed in the article do not necessarily represent the views of the DOE or the U.S. Government. The U.S. Government retains and the publisher, by accepting the article for publication, acknowledges that the U.S. Government retains a nonexclusive, paid-up, irrevocable, worldwide license to publish or reproduce the published form of this work, or allow others to do so, for U.S. Government purposes. 